\title{
AMIC $^{\circledR}$ totalmente artroscópico (AT-AMIC) para 0 tratamento das lesões osteocondrais do tálus
}

\section{All-arthroscopic AMIC ${ }^{\circledR}$ (AT-AMIC) in the treatment of osteochondral talar lesions}

\author{
Tiago Soares Baumfeld ${ }^{4}$, Daniel Soares Baumfeld ${ }^{2}$, Marcelo Pires Prado ${ }^{3}$, Caio Augusto de Souza Nery ${ }^{1}$ \\ 1. Universidade Federal de São Paulo, São Paulo, SP, Brasil. \\ 2. Universidade Federal de Minas Gerais, Belo Horizonte, MG, Brasil. \\ 3. Hospital Israelita Albert Einstein, São Paulo, SP, Brasil. \\ 4. Escola Paulista de Medicina, Universidade Federal de São Paulo, São Paulo, SP, Brasil.
}

\section{RESUMO}

Objetivo: O objetivo deste estudo é relatar os resultados clínicos pós-operatórios dos pacientes submetidos à técnica $\mathrm{AMIC}{ }^{\circledR}$ totalmente realizada através de artroscopia (AT-AMIC ${ }^{\circledR}$ ) combinada à colocação de enxerto ósseo autólogo, quando necessário.

Métodos: Esta é uma série de casos de 17 pacientes consecutivos que foram submetidos à AT-AMIC ${ }^{\circledR}$, entre janeiro de 2016 e abril de 2017. Nove homens e oito mulheres, entre 15 e 67 anos, foram diagnosticados com LOT e submetidos à cirurgia apenas após falha do tratamento conservador de duração mínima de 3 meses. Os pacientes responderam ao escore AOFAS pré-operatório e no último acompanhamento, que variou de 8 a 20 meses.

Resultados: O tamanho médio das LOT foi de 1,16 cm², sendo o "Raikin-4" o local o mais comum (71\%) das lesões. A osteotomia do calcâneo foi o procedimento mais comumente associado, com a cifra de 18\%. O seguimento médio foi de 12,3 meses. O AOFAS médio antes da cirurgia foi de 46,4 pontos, aumentando para 89,5 pontos no último acompanhamento. Essa diferença foi estatisticamente significante ( $p<0,001)$. Não foram observadas complicações em nenhum dos pacientes tratados.

Conclusão: AT-AMIC ${ }^{\circledR}$ é um método de tratamento confiável e reprodutível para as LOT, atingindo altas pontuações clínicas pós-operatórias, com taxa muito baixa de complicações.

Nível de Evidência IV; Estudos Terapêuticos; Série de Casos.

Descritores: Tálus; Artroscopia; Cartilagem; Fraturas de cartilagem.

\section{ABSTRACT}

Objective: The objective of this study was to report the postoperative clinical outcomes of patients undergoing the all-arthroscopic AMIC ${ }^{\circledR}$ technique (AT-AMIC ${ }^{\circledR}$ ) combined with autologous bone graft placement as necessary.

Methods: This was a case series of 17 consecutive patients who underwent AT-AMIC ${ }^{\circledR}$ between January 2016 and April 2017. Nine men and eight women between 15 and 67 years of age were diagnosed with an osteochondral talar lesion (OTL) and underwent surgery only after failure of conservative treatment of at least 3 months. Patients answered the AOFAS scale before surgery and at the final follow-up session, which ranged from 8 to 20 months.

Results: The mean size of the OTLs was $1.16 \mathrm{~cm}^{2}$, and "Raikin-4" was the most common site of lesions (71\%). Calcaneal osteotomy was the most commonly associated procedure (18\%). The mean follow-up period was 12.3 months. The mean AOFAS score before surgery was 46.4 points, rising to 89.5 points at the last follow-up, and this difference was statistically significant $(p<0.001)$. No complications were observed in any of the treated patients.

Conclusion: AT-AMIC ${ }^{\circledR}$ is a reliable and reproducible treatment method for OTLs and achieves high postoperative clinical scores with very low complication rates.

Level of Evidence IV; Therapeutic Studies; Case Series.

Keywords: Talus; Arthroscopy; Cartilage; Fractures, cartilage.

Trabalho realizado no Hospital São Paulo, Escola Paulista de Medicina, Universidade Federal de São Paulo, São Paulo, SP, Brasil.

Correspondência: Tiago Soares Baumfeld. Rua dos Pampas, 990, Prado - Belo Horizonte, MG, Brasil - CEP: 30411-030. E-mail: tiago.baumfeld@gmail.com Conflito de interesses: Caio Nery é consultor/palestrante para Arthrex, USA. Fonte de financiamento: não há

Data de Recebimento: 06/03/2018. Data de Aceite: 26/03/2018. Online em: 25/05/2018.

\section{(c) (1)}


Como citar esse artigo: Baumfeld TS, Baumfeld DS, Prado MP, Nery CAS. AMIC ${ }^{\circledR}$ totalmente artroscópico (AT-AMIC) para o tratamento das lesões osteocondrais do tálus. Sci J Foot Ankle. 2018;12(2):96-101.

\section{INTRODUÇÃO}

As lesões osteocondrais do tálus (LOT) são entidades patológicas que afetam a cartilagem articular e o osso subcondral. Podem ocorrer em até 50\% dos entorses e fraturas agudas do tornozelo, particularmente em associação com lesões esportivas ${ }^{(1)}$. Vários procedimentos cirúrgicos têm sido usados para tratar as LOT, como o desbridamento e microfraturas ósseas subcondrais, transferência de autoenxerto osteocondral (OATS) e implantação autóloga de condrócitos $(\mathrm{ACl})$, entre outros ${ }^{(2)}$. Embora vários estudos relatem resultados pós-operatórios positivos para essas técnicas, elas estão associadas a várias desvantagens ${ }^{(3-5)}$.

Entre as novas alternativas para tratar essas lesões, a Condrogênese Autóloga Induzida por Matriz (AMIC ${ }^{\circledR}$ ) provou fornecer resultados satisfatórios em um seguimento de médio prazo ${ }^{(6)}$. AMIC ${ }^{\circledR}$ combina microfraturas com a aplicação de Chondro-Gide ${ }^{\circledR}$, uma matriz de dupla camada de colágeno tipo I/III (Geistlich Surgery, Wolhusen, Suíça), usada para estabilizar e proteger o coágulo sanguíneo que resulta das microfraturas do osso subcondral. Além disso, as membranas de colágeno demonstraram ser um bom biomaterial para restaurar o osso subcondral, que é um passo crítico para a regeneração de uma cartilagem hialina saudável( ${ }^{(7)}$.

Inicialmente, esse procedimento foi realizado apenas com uma abordagem aberta, mas recentemente foi descrita a técnica $\mathrm{AMIC}{ }^{\circledR}$ totalmente artroscópica $\left(\mathrm{AT}^{-}-\mathrm{AMIC}^{\circledR}\right)^{(8)}$. Essa abordagem tem as vantagens das incisões menores, menor dissecação de partes moles, melhor visualização articular e melhor recuperação do paciente. Também permite ao cirurgião preencher um possível defeito subcondral com enxerto ósseo autólogo, em apenas um procedimento ${ }^{(9)}$.

O objetivo deste estudo é relatar os resultados clínicos pós-operatórios de pacientes submetidos à técnica AT-AMIC e a colocação de enxerto ósseo autólogo, quando necessário, para o tratamento das lesões osteocondrais do tálus.

\section{MÉTODOS}

Este trabalho foi aprovado pelo Comitê de Ética em Pesquisa com registro na Plataforma Brasil sob o número do CAAE: 80854417.7.0000.5505.

Esta é uma série de casos de 17 pacientes consecutivos que foram submetidos à AT-AMIC ${ }^{\circledR}$, entre janeiro de 2016 e abril de 2017. Nove homens e oito mulheres, entre 15 e 67 anos, foram diagnosticados com LOT com história típica de dor profunda no tornozelo e lesão correspondente na ressonância magnética. A cirurgia foi proposta apenas após falha do tratamento conservador por pelo menos 3 meses. Os pacientes responderam ao escore AOFAS no pré-operatório e no último acompanhamento, variando de 8 a 18 meses. Todos os pacientes responderam o termo de consentimento livre e esclarecido para serem incluídos no estudo. Este estudo atendeu todos os requisitos em relação aos direitos humanos e foi aprovado pelo comitê de ética de nossa instituição.

A análise estatística foi realizada com o software GRETL (2017c). O teste t de Student foi utilizado para comparar dados pré-operatórios e pós-operatórios. Neste estudo, adotamos o valor de 0,05 para o erro alfa e consequente rejeição da hipótese de nulidade.

\section{Técnica cirúrgica}

Todas as intervenções cirúrgicas foram realizadas utilizando a técnica AT-AMIC ${ }^{\circledR(8)}$. A artroscopia anterior do tornozelo foi realizada (Figura 1), sempre que necessário, com a ajuda de um afastador tipo Hintermann ${ }^{\mathrm{TM}}$ (Integra LifeSciences, Plainsboro, NJ), permitindo a adequada distração articular (Figura 2). Após a identificação e desbridamento dos tecidos desvitalizados nas bordas e fundo da lesão osteocondral (Figura 3), foram realizadas microfraturas usando

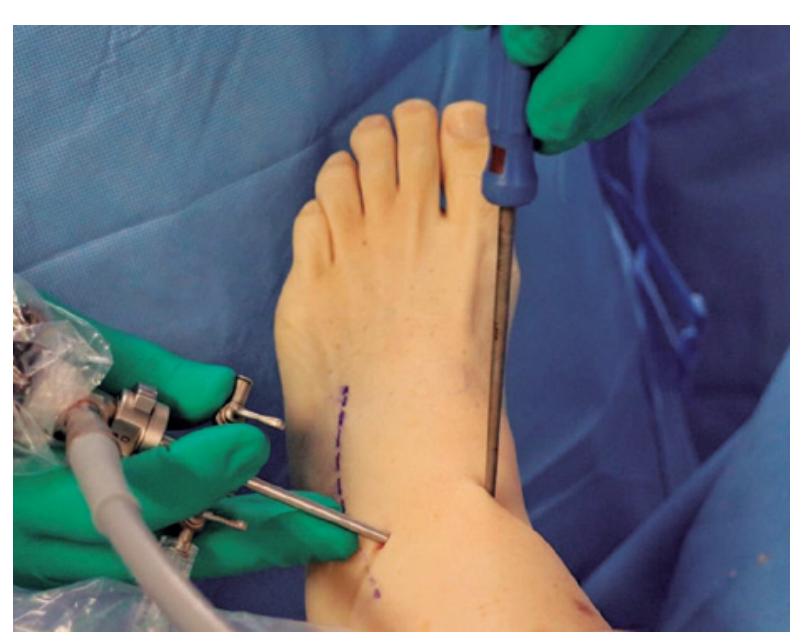

Figura 1. Artroscopia anterior do tornozelo. Fonte: Arquivo pessoal do autor. 
o instrumental Chondro Pick (Arthrex, Naples, FL) que permite a perfuração segura da placa óssea subcondral localizada na camada mais profunda do defeito. Quando constatada a existência de irregularidades ou cistos subcondrais no fundo das lesões, procedemos ao preenchimento e regularização desses defeitos através da enxertia de osso esponjoso autólogo, retirado do corpo calcâneo ou metáfises tibiais ipsilaterais através de uma pequena abordagem percutânea. No momento da introdução e compactação do enxerto de osso esponjoso no fundo das lesões, interrompeu-se o fluxo de soro no interior da articulação passando à condição de artroscopia seca. Foram introduzidas

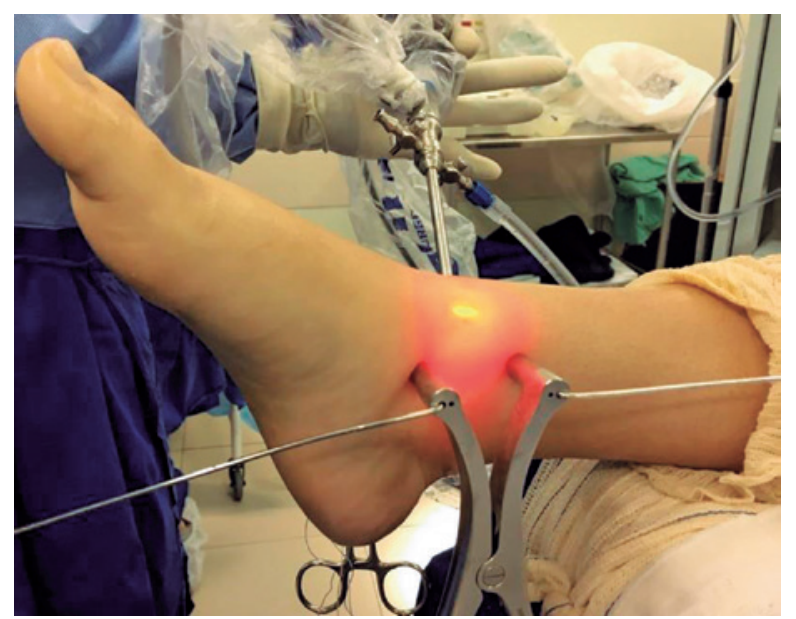

Figura 2. Distrator de Hintermann ${ }^{\mathrm{TM}}$ que permite a distração articular.

Fonte: Arquivo pessoal do autor.

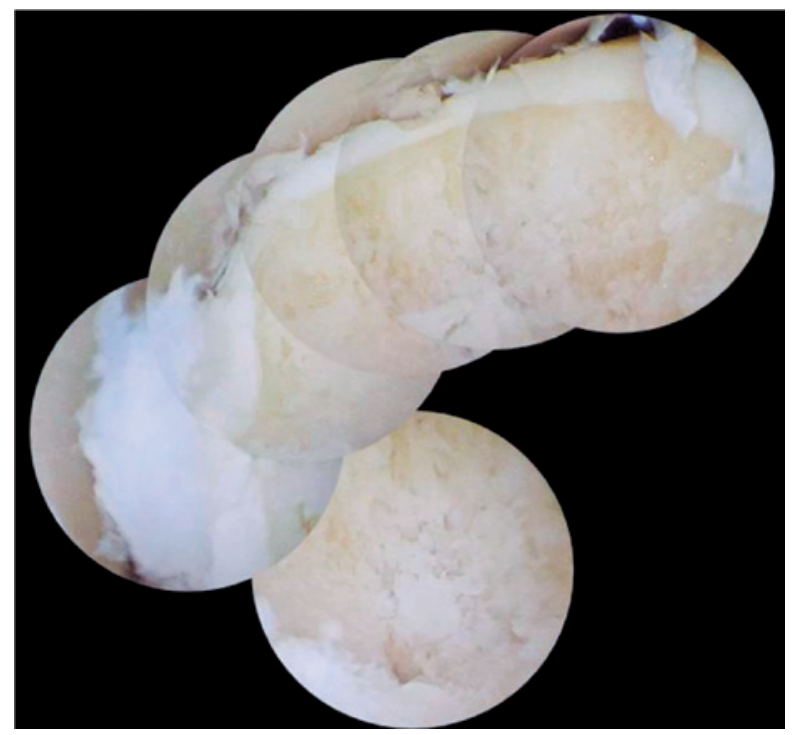

Figura 3. Imagem composta da LOT após o desbridamento. Fonte: Arquivo pessoal do autor. pequenas quantidades de osso esponjoso com a utilização de uma cânula e um trocater. Após a impacção do enxerto, realizamos sua fixação através da aplicação de uma fina camada de cola de fibrina (Tisseel ${ }^{\circledR}$, Baxter, EUA) (Figura 4). 0 formato aproximado e as dimensões da lesão foram cuidadosamente mensurados com o auxílio do probe artroscópico graduado, criando-se um "molde" para a preparação e o corte da membrana colágena. É imperativo assinalar com a caneta cirúrgica a face visceral da membrana de forma a garantir que durante seu posicionamento sobre a lesão, a face visceral esteja voltada para baixo (Figura 5). Uma vez que a membrana esteja adequadamente posicionada de forma a cobrir toda a superfície da lesão, procedemos à sua colagem cuidadosa usando a mesma cola de fibrina mencionada anteriormente (Figura 6). Nesse momento, remove-se o distrator Hintermann ${ }^{\text {TM }}$ permitindo o contato das superfícies articulares que vão estabilizar a membrana em seu lugar adequado. Depois de alguns minutos sem qualquer movimentação do tornozelo, realiza-se a sutura dos portais artroscópicos e a colocação da imobilização pós-operatória. Todos os pacientes foram mantidos com tala gessada suropodálica por 15 dias após a cirurgia, para evitar a mobilização da matriz e, em seguida, foram autorizados a usar uma bota de plástica e a iniciar a movimentação ativa e passiva do tornozelo operado. Os pacientes foram mantidos sem carga de 6 a 8 semanas.

\section{RESULTADOS}

A Tabela 1 descreve os dados demográficos dos 17 pacientes. Foram abordados nove tornozelos esquerdos e oito direitos. $O$ tamanho médio das LOT foi de $1,16 \mathrm{~cm}^{2}$, sendo o
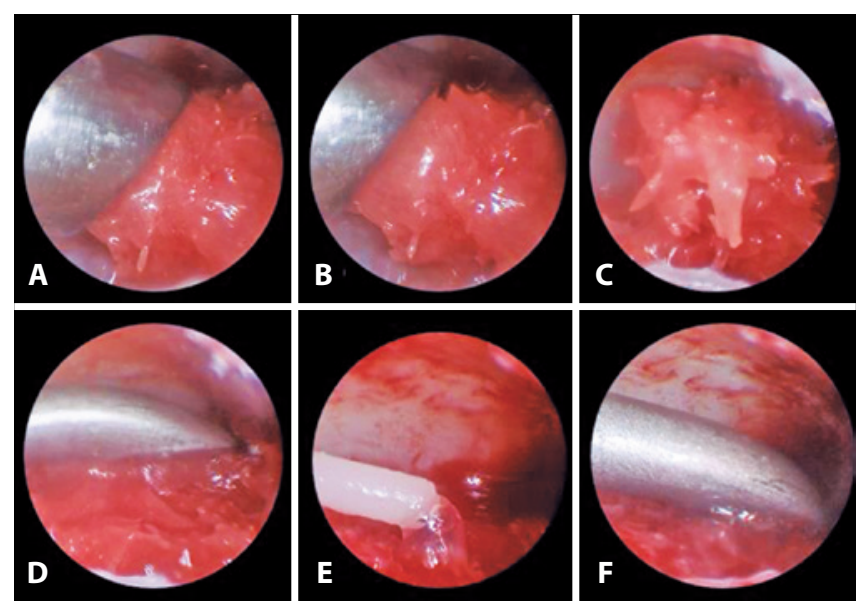

Figura 4. A a C. Osso esponjoso cobrindo o defeito. D. Impactação do osso esponjoso. E a F. Fixação com cola de fibrina. Fonte: Arquivo pessoal do autor. 


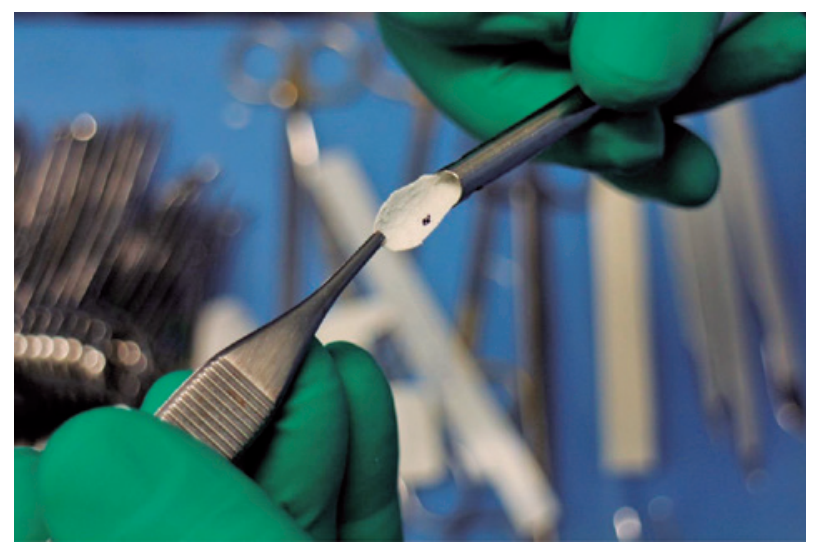

Figura 5. Chondro-Gide ${ }^{\circledR}$ recortado e marcado com uma caneta cirúrgica em sua face visceral.

Fonte: Arquivo pessoal do autor.

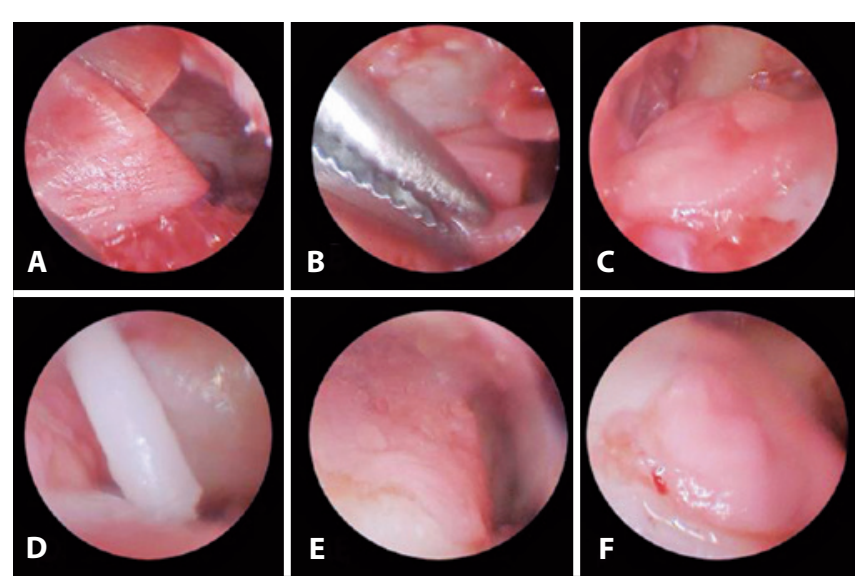

Figura 6. A a C. Posicionamento da membrana Chondro-Gide ${ }^{\circledR}$. $\mathrm{D}$ a E. Fixação com cola de fibrina. F. Aspecto final da construção Fonte: Arquivo pessoal do autor.

"Raikin-4" o local mais comum (71\%) do aparecimento das lesões. A osteotomia valgizante do calcâneo (Dwyer) para o tratamento do varismo do retropé foi o procedimento mais comumente associado ao tratamento das LOT, aparecendo em $18 \%$ dos casos. O seguimento médio foi de 12,3 meses.

O escore AOFAS médio pré-operatório foi de 46,4 , aumentando para 89,5 no último seguimento após a cirurgia. Essa diferença foi estatisticamente significante com um valor de $p<0,001$.

Não foram observadas complicações e não foram necessárias mudanças no protocolo pós-operatório nos pacientes deste estudo.

\section{DISCUSSÃO}

O objetivo do tratamento da LOT é criar um ambiente saudável para a regeneração da cartilagem, eliminar a dor e restaurar a função do tornozelo. Muitas técnicas foram descritas almejando esses objetivos, tais como a estimulação da medula óssea, a implantação de condrócitos autólogos e o aloenxerto de cartilagem juvenil particulada ${ }^{(10)}$. O fibrocolágeno oriundo da microfratura é composto principalmente de colágeno tipo I e foi descrito com propriedades biológicas e biomecânicas inferiores em comparação com a cartilagem hialina normal ${ }^{(11,12)}$. Além disso, a microfratura tem bons resultados em curto prazo, mas com resultados clínicos inferiores em estudos com maior seguimento, em pacientes mais velhos, em lesões profundas e em lesões dos quadrantes centrais ${ }^{(13)}$.

Para resolver esses problemas, a $\mathrm{AMIC}^{\circledR}$ foi utilizada com resultados clínicos e radiológicos muito satisfatórios no joelho, no quadril e no tornozelo, assim como o implante de condrócitos autólogos na matriz (MACl), uma técnica de reparo de cartilagem mais complexa e dispendiosa baseada em células ${ }^{(14,15)}$. Com um acompanhamento mínimo de dois anos, Walther et al., Valderrabano e outros autores relataram excelentes resultados clínicos e radiológicos usando $A M I C{ }^{\circledR}$, atingindo altas pontuações AOFAS e MOCART ${ }^{(16,17)}$. Em um estudo de coorte prospectivo muito recente, Gottschalk et al. corroboram esses achados, atingindo alta satisfação clínica em um seguimento de 5 anos, o seguimento mais longo da literatura ${ }^{(18)}$. Os maiores benefícios da técnica foram observados no primeiro ano; no entanto, a satisfação clínica adicional foi atingida após 5 anos.

Tradicionalmente, a técnica AMIC ${ }^{\circledR}$ é aplicada usando uma osteotomia de maléolo medial ou uma miniartrotomia ${ }^{(19)}$. A osteotomia do maléolo medial aumenta a morbidade do tratamento e tem algumas desvantagens importantes tais como o risco de lesões em estruturas adjacentes, risco de atraso de consolidação ou pseudoartrose e degeneração articular em longo prazo, induzindo ou aumentando a possibilidade do surgimento de osteoartrite ${ }^{(20)}$.

AT-AMIC ${ }^{\circledR}$ é uma abordagem minimamente invasiva que permite ao cirurgião ter os benefícios da terapia à base de membrana sem prejudicar os tecidos moles, tendo, no entanto, as complicações da artroscopia anterior do tornozelo, que variam de 3,4 a 9,0\% ${ }^{(21)}$. Essa técnica foi descrita e usada no joelho, ombro, quadril e tornozelo com resultados encorajadores ${ }^{(22-25)}$. Em 2018, Usuelli et al. publicaram os primeiros resultados de médio prazo da técnica AT-AMIC ${ }^{\circledR}$ para o tálus, com um acompanhamento mínimo de 24 meses. Os vinte pacientes atingiram uma pontuação média no AOFAS de 86.6 (dentro da boa média de resultados da literatura) e um escore MOCART médio de 50.9 no seguimento final. $O$ autor sugere que os cirurgiões do pé e do tornozelo devem adotar a técnica AT-AMIC ${ }^{\circledR}$ para o tratamento das LOT de- 
Tabela 1. Dados dos pacientes

\begin{tabular}{|c|c|c|c|c|c|c|c|c|c|}
\hline № do paciente & Lado & Idade & Sexo & AOFAS PRÉ & AOFAS PÓS & Seguimento & Tamanho $\left(\mathrm{cm}^{2}\right)$ & Local Raikin & Procedimento associado \\
\hline 1 & $\mathrm{D}$ & 23 & M & 68 & 86 & 8 & 1.13 & 9 & - \\
\hline 2 & E & 30 & M & 68 & 90 & 8 & 1.09 & 9 & - \\
\hline 3 & $\mathrm{D}$ & 30 & M & 28 & 87 & 16 & 2.35 & 5 & $\begin{array}{l}\text { Estabilização da } \\
\text { sindesmose }\end{array}$ \\
\hline 4 & $\mathrm{D}$ & 34 & M & 32 & 85 & 9 & 1.02 & 7 & - \\
\hline 5 & E & 32 & $\mathrm{~F}$ & 71 & 100 & 18 & 0.62 & 7 & - \\
\hline 6 & $\mathrm{D}$ & 21 & $\mathrm{~F}$ & 52 & 90 & 17 & 2.07 & $4,5,6,7$ & Brostrom artroscópico \\
\hline 7 & $\mathrm{D}$ & 37 & $\mathrm{~F}$ & 33 & 90 & 14 & 1.33 & $4,5,6,7$ & Osteotomia do Calcâneo \\
\hline 8 & $\mathrm{D}$ & 46 & $\mathrm{~F}$ & 44 & 86 & 13 & 0.75 & $1,2,3,4$ & Artrolise \\
\hline 9 & $E$ & 48 & M & 60 & 92 & 20 & 3.41 & $1,4,7$ & $\begin{array}{l}\text { Osteotomia do calcâneo + } \\
\text { impacto anterior }\end{array}$ \\
\hline 10 & E & 67 & $\mathrm{~F}$ & 59 & 86 & 10 & 0.69 & 4 & Osteotomia do Calcâneo \\
\hline 11 & $\mathrm{E}$ & 60 & $\mathrm{~F}$ & 36 & 82 & 14 & 0.88 & $4,5,6,7$ & - \\
\hline 12 & $\mathrm{D}$ & 67 & $\mathrm{~F}$ & 34 & 87 & 14 & 0.82 & $4,5,6,7$ & - \\
\hline 13 & E & 19 & M & 37 & 97 & 11 & 0.61 & 4 & - \\
\hline 14 & E & 44 & M & 42 & 85 & 11 & 0.79 & 4 & - \\
\hline 15 & $\mathrm{D}$ & 15 & M & 46 & 96 & 9 & 0.63 & $4,5,6,7$ & - \\
\hline 16 & $\mathrm{E}$ & 30 & $\mathrm{M}$ & 38 & 93 & 9 & 0.66 & $1,2,3,4$ & - \\
\hline 17 & $E$ & 35 & $\mathrm{~F}$ & 40 & 90 & 8 & 0.86 & 4 & $\therefore$ \\
\hline Média & & 37.5 & & 46.4 & 89.5 & 12.3 & 1.16 & & \\
\hline
\end{tabular}

Fonte: Elaborado pelo autor com base nos resultados da pesquisa.

vido ao baixo risco de complicações, à eficácia e à abordagem minimamente invasiva ${ }^{(25)}$.

Nossa série corrobora com os estudos anteriores ao mostrar que o AT-AMIC ${ }^{\circledR}$ pode atingir resultados muito satisfatórios, pois nossos pacientes alcançaram um índice AOFAS médio de 89.5 no seguimento final, sem complicações observadas nesse ainda curto período de observação.

O achado mais importante deste estudo é que o AT-AMIC ${ }^{\circledR}$ é um método de tratamento confiável e reprodutível para LOT, atingindo altas pontuações clínicas pós-operatórias, sem complicações. As desvantagens deste estudo são a metodologia retrospectiva, sem grupo controle, a falta de avaliação radiológica do tecido reparador e a falta de observação do tempo cirúrgico e retorno à atividade esportiva.

\section{CONCLUSÃO}

AT-AMIC ${ }^{\circledR}$ é um método de tratamento confiável e reprodutível para as LOT, atingindo altas pontuações clínicas estudo. São necessários mais estudos, com metodologia prospectiva para provar sua eficácia.

Contribuição de autores: Cada autor contribuiu individual e significantemente para o desenvolvimento deste artigo: TSB (https://orcid.org/0000-00019244-5194)* concebeu e planejou as atividades que levaram ao estudo, interpretou resultados do estudo, redação do artigo e aprovou a versão final; DSB (https://orcid.org/0000-0001-5404-2132)*: concebeu e planejou as atividades que levaram ao estudo, realizou as cirurgias, participou no processo de revisão e aprovou a versão final; MPP (https://orcid.org/0000-0003-3812-9320)* realizou as cirurgias, participou no processo de revisão e aprovou a versão final; CASN (https://orcid.org/0000-0002-9286-1750)* concebeu e planejou as atividades que levaram ao estudo, realizou as cirurgias, participou no processo de revisão e aprovou a versão final. *ORCID (Open Researcher and Contributor ID).

\section{REFERÊNCIAS}

1. Savage-Elliott I, Ross KA, Smyth NA, Murawski CD, Kennedy JG. Osteochondral lesions of the talus: a current concepts review and evidence-based treatment paradigm. Foot Ankle Spec. 2014;7(5): 414-22.

2. Kraeutler MJ, Chahla J, Dean CS, Mitchell JJ, Santini-Araujo MG, Pinney SJ, Pascual-Garrido C. Current concepts review update. Foot Ankle Int. 2017;38(3):331-42.
3. Giannini S, Buda R, Faldini C, Vannini F, Bevoni R, Grandi G, Grigolo $B$, Berti L. Surgical treatment of osteochondral lesions of the talus in young active patients. J Bone Joint Surg Am. 2005;87(Suppl 2): 28-41.

4. Knutsen G, Drogset JO, Engebretsen L, GrøntvedtT, Isaksen V, Ludvigsen TC et al. A randomized trial comparing autologous chondrocyte implantation with microfracture: findings at five years. J Bone Joint Surg Am. 2007;89(10):2105-12. 
5. Schneider TE, Karaikudi S. Matrix-induced autologous chondrocyte implantation (MACl) grafting for osteochondral lesions of the talus. Foot Ankle Int. 2009;30(9):810-4.

6. Gottschalk O, Altenberger S, Baumbach S, Kriegelstein S, Dreyer F, Mehlhorn $A$, et al. Functional medium-term results after autologous matrix-induced chondrogenesis for osteochondral lesions of the talus: a 5-year prospective cohort study. J Foot Ankle Surg. 2017; 56(5):930-936

7. Cavallo M, Buda R, Vannini F, Castagnini F, Ruffilli A, Giannini S Subchondral bone regenerative effect of two different biomaterials in the same patient. Case Rep Orthop. 2013;2013:850502.

8. Usuelli FG, de Girolamo L, Grassi M, D’Ambrosi R, Montrasio UA, Boga M. All-arthroscopic autologous matrix-induced chondrogenesis for the treatment of osteochondral lesions of the talus. Arthrosc tech. 2015;4(3):e255-9.

9. D'Ambrosi R, Maccario C, Ursino C, Serra N, Usuelli FG. Combining Microfractures, Autologous bone graft, and autologous matrix-induced chondrogenesis for the treatment of juvenile osteochondral talar lesions. Foot Ankle Int. 2017;38(5):485-495.

10. Grambart ST. Arthroscopic management of osteochondral lesions of the talus. Clin Podiatr Med Surg. 2016;33(4):521-30.

11. Murawski CD, Foo LF, Kennedy JG. A review of arthroscopic bone marrow stimulation techniques of the talus: the good, the bad, and the causes for concern. Cartilage. 2010;1(2):137-44.

12. Nehrer S, Spector M, Minas T. Histologic analysis of tissue after failed cartilage repair procedures. Clin Orthop Relat Res. 1999;(365):149-62.

13. Yoshimura I, Kanazawa K, Takeyama A, Angthong C, Ida T, Hagio T, et al. Arthroscopic bone marrow stimulation techniques for osteochondral lesions of the talus: prognostic factors for small lesions. Am J Sports Med. 2013;41(3):528-34.

14. Giannini S, Buda R, Ruffilli A, Cavallo M, Pagliazzi G, Bulzamini MC, et al. Arthroscopic autologous chondrocyte implantation in the ankle joint. Knee Surg Sports Traumatol Arthrosc. 2014;22(6):1311-9.

15. Mancini D, Fontana A. Five-year results of arthroscopic techniques for the treatment of acetabular chondral lesions in femoroacetabular impingement. Int Orthop. 2014;38(10):2057-64.

16. Valderrabano V, Miska M, Leumann A, Wiewiorski M. Reconstruction of osteochondral lesions of the talus with autologous spongiosa grafts and autologous matrix-induced chondrogenesis. Am J Sports Med. 2013;41(3):519-27.

17. Walther M, Martin K. Scaffold based reconstruction of focal full thickness talar cartilage defects. Clin Res Foot Ankle. 2013;1(2):115.

18. Gottschalk O, Altenberger S, Baumbach S, Kriegelstein S, Dreyer F, Mehlhorn A, et al. Functional medium-term results after autologous matrix-induced chondrogenesis for osteochondral lesions of the talus: a 5-year prospective cohort study. J Foot Ankle Surg. 2017;56(5):930-6.

19. Walther M, Altenberger S, Kriegelstein S, Volkering C, Röser A. Reconstruction of focal cartilage defects in the talus with miniarthrotomy and collagen matrix. Oper Orthop Traumatol. 2014; 26(6):603-10.

20. Kreuz PC, Steinwachs M, Edlich M, Kaiser T, Mika J, Lahm A, Südkamp N. The anterior approach for the treatment of posterior osteochondral lesions of the talus: comparison of different surgical techniques. Arch Orthop Trauma Surg. 2006;126(4):241-6.

21. Ferkel RD, Small HN, Gittins JE. Complications in foot and ankle arthroscopy. Clin Orthop Relat Res. 2001;(391):89-104.

22. PiontekT, Ciemniewska-Gorzela K, Szulc A, Naczk J, Słomczykowski M. All-arthroscopic AMIC procedure for repair of cartilage defects of the knee. Knee Surg Sports Traumatol Arthrosc. 2012;20(5):922-5.

23. Fontana A. Autologous Membrane Induced Chondrogenesis (AMIC) for the treatment of acetabular chondral defect. Muscles Ligaments Tendons J. 2016;6(3):367-71.

24. Cuéllar A, Ruiz-lbán MÁ, Cuéllar R. The use of all-arthroscopic autologous matrix-induced chondrogenesis for the management of humeral and glenoid chondral defects in the shoulder. Arthrosc Tech. 2016; 5(2):e223-7.

25. Usuelli FG, D'Ambrosi R, Maccario C, Boga M, de Girolamo L. All All-arthroscopic AMIC ${ }^{\circledR}$ (AT-AMIC ${ }^{\circledR}$ ) technique with autologous bone graft for talar osteochondral defects: clinical and radiological results. Knee Surg Sports Traumatol Arthrosc. 2018;26(3):875-81. 